\title{
La evaluación de la intervención comunitaria: un marco para la reflexión
}

\author{
Belén Pascual Barrio \\ Universitat de Les ILles Balears
}

\section{Resumen}

En este artículo se presenta la propuesta de un marco reflex i vo para la evaluación que integra aquellos aspectos, variables y condicionantes que influyen en la intervención socioeducativa y deben ser considerados para la mejora de la evaluación de la misma. Las sugerencias que se plantean parten de la experiencia de evaluación externa de un plan de desarrollo comunitario: la Iniciativa Comunitaria Urban-El Temple de Palma de Mallorca, que se realizó entre los años 1997 y 2001 y, de forma específica, de los programas de inserción para el empleo.

Palabras Clave: for mación ocupacional, desarrollo comunitario, inserción laboral, intervención socioeducativa mercado laboral, prevención, evaluación.

\section{Summary}

In this article we propose a theoretical model to make a global assessment of all the dimensions, variables and conditions that have to be considered to improve the assessment itself. These proposals came from our experience making the external assessment of a social community development experience: the El Temple-Urban Community Initiative in Palma de Mallorca that took place between 1997 and 2001 and, more specifically, socioemployment insertion programmes.

KEYWORDS: vocational training, social community development, labour insertion, socioeducational intervention, prevention, assessment.

A menudo existen fisuras entre aquellos principios que fundamentan la intervención comunitaria y aquellos otros que justifican la evaluación de la misma. Si bien el salvar esta distancia resulta complejo, el reconocimiento de la misma representa un potencial de mejora de la intervención y de la evaluación. Consideramos que sólo la declaración de estas fisuras permite plantear nuevas estrategias que respeten los principios de la intervención comunitaria que, no por difíciles de llevar a la práctica, dejan de ser relevantes. De hecho, el evaluador no puede desatender esas posibles contradicciones inherentes a la evaluación.

El proceso de intervención social es comunitario en cuanto que supone la actuación en un entorno o contexto definido en el que se dan problemas concretos y compartidos y conlleva la alteración de las relaciones entre 
individuos, grupos, asociaciones e instituciones. En este proceso se pretende que la persona no sólo sea objeto de intervención sino también sujeto activo, participante y protagonista de la intervención. Marchioni (1969) insiste en afirmar que las intervenciones técnicas, aun siendo fundamentales, deben siempre estar orientadas y dirigidas por la acción social de base que es, en definitiva, la que puede conseguir una efectiva penetración que facilite el cambio real.

Cada modelo de desarrollo comunitario es diferente y debe partir de las necesidades y demandas específicas del territorio de referencia (Marchioni, A. M., 2001) y contar con la integración y aprove chamiento de todos los recursos existentes. De hecho, uno de los principios fundamentales de la intervención comunitaria consiste en la adecuación a las necesidades de la propia comunidad. La justificación de la intervención conlleva la participación activa de la comunidad, así como el ajuste de la acción a los valores y capacidades potenciales de ésta.

Desde esta perspectiva, el desarrollo comunitario implica el esfuerzo conjunto entre poderes públicos y comunidad, la correspondencia de intereses y la coordinación y la comunicación interna entre los diferentes sectores de la intervención. La relación con los diversos agentes sociales y el protagonismo de éstos se convierten en requisitos imprescindibles, pudiendo partir la iniciativa comunitaria de cualquiera de ellos: la administración municipal, los técnicos de los diferentes servicios (tanto públicos como privados) y la sociedad civil (población y organizaciones/asociaciones).

La propuesta reflexiva que plantemos en este artículo parte de la experiencia de evaluación externa de una iniciativa de desarrollo comunitario llevada a cabo en Palma de Mallorca: la Iniciativa Comunitaria Urban-El Temple y, de forma específica, de sus programas de inserción para el empleo. Esta Iniciativa, promovida por el Ayuntamiento de Palma de Mallorca, se desarrolló entre los años 1997 y 2001 en los barrios de Sa Gerreria, La Calatrava y El Temple. En esta zona la precariedad social se concentraba en hogares y espacios localizados, principalmente en el barrio de Sa Gerreria. El diseño de la iniciativa fue elaborado por un equipo multidisciplinar, compuesto por representantes de diferentes áreas municipales que colaboraron con el Patronato de la Vivienda. Desde la iniciativa Urban se planteó un plan conjunto de intervención urbanística, social, laboral y educativa. La oferta formativa consistió en programas de alfabetización de adultos, formación profesional, educación para el tiempo libre y animación sociocultural. En concreto, el programa de Formación e inserción laboral contó con proyectos de formación ocupacional, programas de inserción laboral, cursos de reciclaje y perfeccionamiento profesional, programas de orientación profesional y cursos de salud laboral.

Como complemento a la evaluación continua que realizaban los profesionales de estos programas, se encargó una evaluación externa al Departamento de Ciencias de la Educación de la Universitat de les Illes Balears. A lo largo del proceso de intervención se hizo seguimiento de los proyectos con el fin de valorar los cambios y los efectos que iban produciéndose en cada una de las fases. La autora de este artículo participó en el equipo de evaluación externa y continuó investigando sobre esta temática, presentando finalmente su tesis doctoral en julio de 2004, con el siguiente título: "La evaluación de programas de intervención socioeducativa. La experiencia de Urban-El Temple de Palma de Mallorca."

\section{La evaluación de la intervención comunitaria: retos y riesgos}

En la intervención comunitaria es fundamental la consideración de la propia comunidad, las unidades de convivencia y las redes sociales, sin embargo, suele darse con fre- 
cuencia la asistencia puntual y las medidas de carácter paternalista en lugar de estrategias participativas que respondan a necesidades reales y den respuesta directa a las demandas de los interesados. Partir de un análisis de necesidades desde un enfoque generalista y sin tener en cuenta la comunidad y el entorno sociocultural de referencia nos aleja del conocimiento riguroso de las potencialidades y los obstáculos que puedan darse en la intervención.

En este sentido, la evaluación puede ayudarnos a identificar los aspectos positivos y negat i vos de la intervención, los diferentes elementos que contribuyen al logro de los objetivos y puede orientarnos hacia la mejora de la práctica. Desde este punto de vista, la e valuación se orienta a la toma de decisiones para la mejora de la calidad de la intervención constituyendo un instrumento que ayuda a conocer a fondo el funcionamiento de los programas de intervención, de las organizaciones y de los profesionales que los llevan a término. A partir de la recogida sistemática de información podemos ampliar el conocimiento sobre las necesidades en sus contextos, sobre el nivel de calidad de los programas y sobre su adecuación y coherencia con respecto a las necesidades reales. Asimismo, podemos conocer el tipo de planificación, los recursos disponibles, el seguimiento de la puesta en marcha y aplicación de los programas, los resultados conseguidos y el impacto provocado.

Partiendo de la posibilidad de mejora de las prácticas, la evaluación puede considerarse como un sistema de control al servicio de los organismos o instituciones contratantes o como un instrumento de cambio que permite mejorar la calidad de vida, en el sentido más amplio, de todos los implicados en el proceso. La evaluación, vista como un instrumento de mejora y optimización de la acción social, se convierte en un elemento de cambio ya que representa tanto los intereses de la institución y de los pro- fesionales, como también de los usuarios y del conjunto de la ciudadanía (Gómez, 2004: 210-217). En todo proceso de evaluación de programas de intervención socioeducativa existe una relación dialéctica entre las dimensiones de control y de cambio social, aunque una de ellas puede destacar sobre la otra de acuerdo con el tipo de aplicación de la evaluación que se trate. Pero, a menudo, la función de control se antepone a la de cambio debido a una concepción excesivamente burocratizada de los programas que, al margen de la participación de la comunidad, cede su responsabilidad exclusivamen te a políticos y técnicos.

Atendiendo a la clasificación de Marín y Medina (1995) sobre las dificultades propias de la evaluación y elaboración de los programas de intervención socioeducativa, pode mos considerar que uno de los principales riesgos es la falta de un análisis suficientemente concreto sobre las necesidades del colectivo al que se dirigen los programas y, como consecuencia, la falta de diseños iniciales suficientemente operativos que permitan evaluar los procesos y los resultados de una forma rigurosa.

La ausencia de una perspectiva comunitaria en los procesos de planificación y evaluación se produce cuando los planificadores, ejecutores y evaluadores de la intervención acaparan protagonismo y actúan al margen de la comunidad adoptando un enfoque excesivamente externo (limitado a la definición desde fuera del contexto). Esta forma de intervenir y evaluar favorece planteamientos excesivamente abstractos que centran su atención sobre ciertos sectores sociales y no sobre el sistema social, ocultando el carácter"políti camente determinado" de muchas de estas necesidades y presentándolas como demandas de los propios afectados. Mientras tanto, no se analiza el papel de las propias instituciones de servicios sociales en la configuración de su demanda (De Prada et al., 2003). De hecho, a menudo en la evaluación no se 
asume la responsabilidad última de la intervención y se atribuye a factores externos el fracaso de la intervención, como las dificultades de partida del colectivo destinatario. Podemos atribuir esa tendencia a que cuando existen fallos tendamos a evaluar a las personas y no las actividades o procesos de intervención. Se trata de la dificultad para reconocer nuestras limitaciones y el exceso de confianza que a veces se deposita en especulaciones teóricas.

La falta de participación de los agentes sociales en la planificación, ejecución y evaluación de la misma hace que no se corresponda el análisis de necesidades a la propuesta de intervención y que la programación no concierna a la acción real, es decir, que se produzca una brecha entre la teoría y la práctica. Desde un enfoque tradicional, se confiaba en que necesariamente las conclusiones obtenidas a partir de la evaluación servirían para la mejora futura de la implementación del programa. Sin embargo, a veces el interés de los responsables de la evaluación puede ser únicamente la justificación formal de una evaluación, quedando al margen todos los aspectos de mejora de la intervención social en la comunidad.

Con el fin de evitar una práctica que responda a la justificación formal, la evaluación actual introduce en los grupos de trabajo a los gestores desde la fase previa, durante la planificación del qué y cómo evaluar, así como en la fase de análisis de los resultados, para fomentar la corresponsabilidad en las conclusiones y, con ello, su futura utilización con vistas al perfeccionamiento del programa (Patton, 1987). De esta forma, la evaluación es más participativa y permite la introducción de mejoras en el proceso. Concretamente, las prácticas que se proponen son las siguientes:

En primer lugar, definir previamente y con el mayor grado de concreción posible el colectivo diana. No conociendo las necesidades locales de forma concreta, los cambios sociales y de inserción resultan difíciles de evaluar. Así pues, se trata de:

- Evitar diagnósticos generalistas.

- Que los objetivos de la intervención se adecuen específicamente a las problemáticas y necesidades sociales detectadas.

En segundo lugar, a partir de diagnósticos operativos se favorece la concreción en el diseño que, partiendo de una problemática dada, tratará de establecer los objetivos a seguir, los aspectos metodológicos y el procedimiento de desarrollo de la evaluación. El diseño debe presentar una estructura y estrategias delimitadas que permitan evaluar durante y al final del proceso. Se debe intentar:

- Evitar diseños en los que se presentan las acciones de forma tan genérica que ésta no puede ser evaluada.

- Que el proyecto se ajuste la política, las necesidades y prioridades locales.

- Que la duración se adecue a las necesidades de los beneficiarios

En tercer luga r, la evaluación como estrategia de mejora constituirá un elemento de cambio y también de control durante el proceso del programa. Desde esta perspectiva, la evaluación permite intervenir ante los obstáculos que van surgiendo.

- La evaluación interna y externa favorecen no sólo la crítica de datos objetivos sino también de la intervención.

- La evaluación continua favorece la retroalimentación durante el proceso.

- La estrategia de la autoevaluación contribuye a un mayor rendimiento, aprendizaje e innovación.

- La implicación de todos los miembros en el proceso de autoevaluación y toma de decisiones, se consideran factores determinantes para el progreso de la calidad de la intervención. 
- En ese seguimiento constante y compromiso de mejora es necesaria la sistematización de objetivos operativos que permitieran valorar determinados aspectos del desarrollo del programa.

- La posibilidad de crear y participar son aspectos que se relacionan con la formación permanente a partir de las necesidades que van surgiendo "sobre la marcha".

- El empleo a lo largo del proceso de la autoevaluación permite la adaptación y mejora de la intervención, la valoración conjunta del equipo y la propuesta de cambios sobre la marcha.

La investigación acción es una metodología que parte de la consideración del propio objeto de estudio (la comunidad) como sujeto activo en el análisis. A lo largo del proceso, los diferentes agentes (representantes de la administración, representantes del tejido asociativo y ciudadanos en general) colaboran a partir de comisiones de seguimiento y grupos de investigación. El carácter flexible de este paradigma permite superar la dicotomía entre teóricos (expertos/técnicos) y prácticos (activistas sociales), facilitando la comunicación interprofesional y el trabajo colectivo.

Esta metodología permite desarrollar el análisis de las necesidades de un territorio con la participación de los propios actores implicados y con la finalidad principal de que las propuestas de mejora favorezcan, efecti vamente, la transformación del entorno, se ajusten más a la realidad concreta y que refuercen las potencialidades del propio colectivo. Los actores implicados se convierten en los protagonistas de un proceso participativo en el que, a través de la palabra y los espacios de reflexión, se mejora el conocimiento del objeto de estudio.

La metodología de la acción participativa se inscribe en el ámbito local y agrupa ámbitos diversos del desarrollo (vivienda, empleo, cultura, salud,...), con objetivos socialmente integradores que se plasman en planes y propuestas de intervención integral en el territorio que contemplen las diferentes realidades. Los ejes centrales en el desarrollo de una Investigación-Acción-Participativa son los siguientes:

- Fase de concreción e identificación de un área problemática por la cual esté afectado un grupo a nivel local.

- Fase de apertura a todos los puntos de vista existentes entorno a la problemática y objetivos definidos: se trata de elaborar un diagnóstico, y de recoger posibles propuestas.

- Fase de negociación de las propuestas y concreción de las mismas en líneas de actuación. - La puesta en marcha de estas actuaciones abre un nuevo ciclo en el que se detectarán nuevos síntomas y problemáticas y en el que cabrá definir nuevos objetivos.

\section{Un esquema para la reflexión sobre la evaluación de la intervención comunitaria}

Con el fin de estructurar esas diferentes fases de la evaluación en un continuo que contengael proceso de intervención en su conjunto, proponemos un instrumento que permita integrar los distintos niveles de análisis teórico en diferentes ámbitos y momentos de la intervención y de la evaluación. Se trata de un esquema orientativo que integra dos variables básicas: el nivel de análisis que apliquemos y el momento en el que nos encontremos. Concretamente, desde estas dos dimensiones, los objetivos serían:

- Detectar los puntos fuertes, débiles, ame nazas o debilidades, considerando distintos niveles y momentos de la evaluación.

- Valorar las consecuencias secundarias de los programas en diversos niveles y momentos.

- Anticipar el efecto de las consecuencias más probables de los mismos desde diversos niveles y momentos. 
En la práctica de la evaluación cabe integrar aquellos aspectos sociales, grupales e individuales de la realidad que envuelven la intervención socioeducativa. Para ello, es necesario considerar tres ámbitos de estudio, ya que cada uno de ellos nos aproxima a una vertiente de la realidad social (Rubio y Varas, 1997). En primer lugar, desde el punto de vista del individuo influido por factores grupales y sociales. En segundo lugar, desde el punto de vista de los grupos que reciben tanto la influencia de las características individuales de sus miembros como de la sociedad en la que se encuentran. En tercer lugar, desde el punto de vista de la sociedad influida por las actuaciones y características de los individuos y grupos.

Partimos de la consideración del sistema social como estructura de relaciones entre elementos, cuya suma no se corresponde con el total. Al analizar e interpretar los problemas sociales o individuales debemos tener en cuenta la interacción de múltiples variables y diferentes ámbitos como factores explica t i vos de estos problemas o situaciones. Así pues, planteamos la propuesta de un modelo para la evaluación que integra aquellos aspectos, variables y condicionantes de la evaluación y la intervención. En primer lugar, el nivel de análisis: macro y micro. En segundo lugar, el tiempo como variable que marcará el momento de referencia para cada una de las fases de la evaluación. Partiendo de la consideración de la intervención comunitaria desde su dimensión dinámica y de proceso, este esquema representa el tiempo y espacios de la evaluación como un continuo estructurado alrededor de dos ejes que delimitan este espacio.

En el centro de la gráfica situamos el programa a evaluar, en el marco de un contexto social, económico y cultural determinado. Centramos nuestro tema de estudio en el nivel intermedio, entre el individual y el social. Los programas de intervención socioeducativa se insertan en el ámbito psicoso-

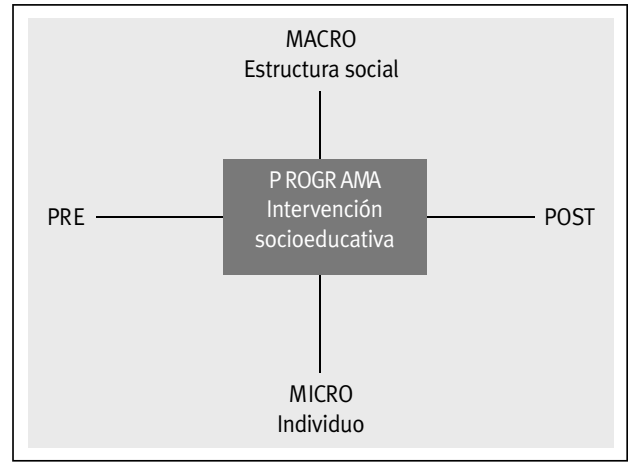

cial y para su análisis se consideran los aspectos del entorno más próximo a la iniciativa de intervención. Es decir, se trata del análisis de las organizaciones o grupos implicados así como la estructura de relaciones entre individuos: sistemas de roles, tareas, comunicación, afectividad, estatus, características individuales y normas. Con el fin de conocer en mayor medida aquellas dimensiones sociales relacionadas a los procesos educativos podríamos distinguir el análisis de cuatro grandes áreas temáticas con respecto al entorno de los programas (Setién, 1993):

- El entorno físico, económico y social del barrio.

- Las características del mercado de trabajo, infraestructuras públicas y privadas.

- Los agentes sociales y económicos generadores de actuaciones, intervenciones y capaces de dinamizar los barrios.

- Las actuaciones públicas de promoción del barrio, programas socioeducativos y económicos dirigidos a erradicar procesos de exclu sión social.

La centralidad del programa en el gráfico no se corresponde con un mayor interés por el análisis psicosocial que por los niveles macro o microsociales. En cualquier caso, ocupa el lugar central por ser nuestro objeto de estudio. Ahora bien, como proceso y espacio abierto al exterior e influido, a su vez, por cada uno de los individuos, grupos e institu- 
ciones participantes, debemos considerar ese centro en expansión en el sentido que nos marcan los dos ejes:

- El eje horizontal representará el tiempo de la evaluación y en los dos extremos situaríamos la evaluación previa y posterior (pre y post).

- El eje vertical representará el nivel de análisis que se adopte en la evaluación y cuyos vértices serían los niveles macro y micro respectivamente.

Con respecto al eje horizontal consideraremos todos los momentos relativos a la intervención desde el inicio de su planteamiento teórico hasta la valoración posterior a la intervención. Se incluyen por tanto todas las fases y modelos de evaluación posibles a lo largo del tiempo. Con respecto al eje vertical, con sideraremos aquellos factores que se extien den desde un nivel macro a un nivel microsocial.

En cuanto al eje vertical, se trata de considerar a lo largo del proceso todos los condicionantes desde la interrelación entre los distintos niveles de análisis.

- Desde el ámbito sociológico (macrosistema) se analizan sociedades y comunidades, la estructura de relaciones entre organiza ciones y grupos: económicas, políticas, laborales, culturales y educativas. Cada grupo o sociedad parte de una visión e interpretación de la realidad, elementos culturales, ideológicos (creencias, valores, opiniones), así como elementos demográficos, económicos, ecológicos.

- Desde el ámbito psicológico (microsistema) se contempla el análisis de los individuos y los contextos familiares. Se trata del sistema psicológico o microsistema, ele mentos que en su interrelación conforman la estructura de la personalidad. Para el análisis a este nivel se considerará la dimensión individual de los cambios, los itinerarios indi- viduales, atendiendo a indicadores psicosociales que nos aproximarán a conceptos como el bienestar subjetivo. En este nivel es más habitual el manejo de instrumentos de evaluación como son las valoraciones diagnósticas, el registro del seguimiento individualizado, tutorías, las evaluaciones de proceso y finales (tanto de aprendizajes, de conocimientos adquiridos, de actitudes, etc.).

Estos ámbitos se contemplan como sistemas integrados entre sí y entre ellos se produce intercambio de información. Recordemos que P. Bourdieu (1988) analiza los mecanismos culturales de diferenciación social y, refiriéndose a las trayectorias de las clases sociales, apunta los dos factores que determinan las mismas. En primer lugar, las fuerzas que estructuran el espacio social y los mecanismos de regulación y reproducción del mismo; en segundo lugar, la inercia del individuo, que viene dada por el conjunto de propiedades (su capital económico y cultural) y por las estrategias que desarrolla.

Para cada uno de los espacios que delimitan los ejes podemos situar aquellos facto res a considerar, tanto desde la posibilidad de cambios como desde los posibles obstáculos que puedan amenazar estos procesos. Por ejemplo, podemos atender a las limitaciones que condicionan la intervención comunitaria como son los factores estructurales económicos y sociales, problemáticas como el desempleo, la marginalidad y la pobreza que afectan a las economías nacionales. A pesar de que lo local ofrece múltiples posibilidades, no podemos perder de vista la existencia de un mercado laboral que excluye a un sector importante de la población (parados de larga duración, trabajadores en precario) y la existencia de nuevas formas de discriminación por razón de sexo, etnia o edad: exclusión de amplios sectores femeninos, inmigrantes internacionales, jóvenes y jubilados sin recursos suficientes.

Desde la política social de los gobiernos se potencia el protagonismo de los ayunta 
mientos por su proximidad a los ciudadanos y su capacidad para detectar las necesidades del entorno, por una parte, y de los ciudadanos, por otra. De acuerdo con Caride (1997) lo local, el pueblo, el barrio son ámbitos privilegiados de democracia de base y para que éstos cumplan coherentemente su papel la intervención (y planificación) debe atender tanto la integralidad como a la concreción en la acción, la adecuación a las necesidades y a las expectativas de cada comunidad.

La política social, siguiendo criterios de descentralización, debe permitir contextuali zar procesos de intervención social sin perder su carácter global, polivalente e integral. Sin embargo, las propuestas de intervención que suponen la revitalización de la comunidad se están produciendo desde unas ex igencias diferentes a las de años atrás, en un momento que se caracteriza por la economización de los recursos estatales. El trabajo social comunitario y el fortalecimiento del tejido social, puede dar respuesta a las demandas sociales que el Estado no puede cubrir. Desde este punto de vista, la comunidad se convierte en un marco más próximo, flexible y eficaz que la Administración Pública (Montagut, T., 1997) y, frente a dinámicas individualistas, se defiende un modelo de ciudadanía basado en la solidaridad, la cooperación y la adquisición de responsabilidades de los individuos con respecto a los problemas de su comunidad.

A pesar de los avances de las últimas tres décadas, la participación directa de los ciudadanos en las cuestiones sociales que les afectan, a menudo, es limitada. El modelo de democracia representativa limita la participación directa de los ciudadanos y otorga una gran parte de las funciones a técnicos y profesionales de la administración pública que, como interlocutores con los ciudadanos, se encargan de diagnosticar las necesidades y proponer acciones de intervención, sin cuestionarse las contradicciones propias del sistema social.
La resolución de los problemas sociales implica la promoción de procesos de cambio a partir de metodologías participativas. En caso contrario, se puede caer en situaciones de inmovilismo que no vayan más allá de la legislación y la regulación normativa (Arellano et al., 1998): "La atención a las necesidades sociales requiere favorecer procesos democráticos de gestión desde abajo, flexibilizando los procesos de intervención, a partir de metodologías participativas y de participación directa". Este tipo de metodologías pueden evitar el inmovilismo o el desarrollo de procesos aparentemente participatives que no responden a las necesidades reales.

Aun así, como cualquier otro tipo de metodología, puede ser utilizada en un sentido diferente al desarrollo humano y por ello, la detección de las contradicciones que puedan darse en la práctica, o el mal uso de esta estrategia, también se convierten en una exigencia del propio proceso de participación. De hecho, ya a finales de los años sesenta se produjo un replanteamiento del desarrollo comunitario y de los modelos de actuación llevados a cabo. A esa reflexión contribuyó la emergencia de nuevos movimientos sociales y el auge del pensamiento sociológico crítico que denunciaba la tendencia a trasladar e implantar modelos de intervención sin adecuarlos a las necesidades reales. A pesar de ese replanteamiento, aún en la actualidad la institucionalización de la intervención comunitaria, aunque desde planteamientos democráticos se alejan de la participación directa de la población (De Prada et al., 2003).

\section{Conclusiones}

Desde las ciencias sociales se hace necesaria una mirada crítica que nos permita plantear y revisar el saber y el hacer comunitarios, planteando opciones congruentes con los

[ 136 ] SIPS - PEDAGOGÍA SOCIAL (ISSN-1139-1723) № 14 TERCERAÉPOCA 
principios de una metodología multidisciplinar y analizando no sólo las posibilidades que ofrecen las propuestas existentes, sino también los aspectos contradictorios, que aunque latentes, puedan amenazar las buenas prácticas. A lo largo de este trabajo hemos tratado una serie de aspectos del planteamiento actual que pueden vulnerar los principios de la intervención comunitaria. Como hemos visto, a menudo, el planteamiento teórico comunitario se enfrenta a una práctica poco favorecedora de ese vínculo con la comunidad. Por ejemplo, al inicio de la intervención, el riesgo de que los responsables de los programas no compartan la detección de necesidades con los agentes sociales relacionados y tomen una postura autosuficiente produciéndose distanciamiento entre lo que se pretende teóricamente y aquello que finalmente se lleva a la práctica.

Por todo lo dicho, podemos concluir afirmando que son muchos los retos y diferentes los niveles desde los que cabe reflex ionar y actuar. Mientras vivimos un proceso de institucionalización y profesionalización de la intervención comunitaria, pretendemos dar protagonismo real a la comunidad en la intervención social. Entre otros aspectos ya tratados anteriormente, la coordinación entre la labor comunitaria y los servicios profesionalizados se convierte en un reto fundamental. La cooperación entre los profesionales y todos aquellos agentes que participan en la intervención debe constituir una práctica efectiva y establecida conjuntamente desde el inicio de la implementación por parte de todos los agentes implicados. Es conveniente que en el diseño de los proyectos se considere explícitamente la coordinación con el resto de los recursos del territorio, estableciendo los mecanismos y espacios de planificación, colaboración y evaluación conjunta.

\section{Referencias bibliográficas}

Arellano et al. (1999): "La investigación acción participativa: entrevista a Tomás Rodriguez Villasante", en Revista Razón y Palabra. Comunicación y Democracia. № 12, Año 3, octubre 1998-enero 1999. (www.cem.itesm.mx)

Bordieu, P. (1988): La distinción. Criterio y bases sociales del gusto. Barcelona: Taurus.

Caride, J. A. (1997):“Acción e intervención comunitarias”. En Petrus A. (coord.), Pedago gía Social. Barcelona: Ariel.

De Prada, M. A., Actis, W; Oereda, C. (2003): Investigación Acción Participativa. Propuesta para un ejercicio activo de la ciudadanía. Colectivo Ioé. Intervención sociológica. www.nodo50.org/ioe

Gómez Serrán, M. (2004): Evaluación de los servicios sociales. Barcelona: Gedisa.

Marchoni, A. M. (1969): Comunidad y desarrollo. Barcelona: Nova Terra.

Marchoni, A. M. (2001): Comunidad, partici pación y desarrollo. Madrid: Editorial Popular.

Marín, M.; Medina, F. J. (1995): Evaluación de programas socioeducativos. Investigación versus evaluación. Universidad de Sevilla. Departamento de Psicología Social. Comunicación presentada en II Congreso de Nuevas Tecnologías de la Información para la Educación. UIB e ICE. Grupo: Práctica e investigación en las nuevas tecnologías de la información.

Montagut, T. (1997): “Servicios sociales y educación social." En Petrus, A. (coord.), Pedagogía Social. Barcelona: Ariel.

Pascual, B. (2004): La evaluación de programas de intervención socioeducativa. La expe- 
riencia de Urban-El Temple de Palma de Mallorca. Tesis doctoral del Departament de Ciències de l'Educació. Universitat de les Illes Balears.

Patton (1987): How to use qualitative methods in evaluation. Beberly Hills: Sage

Rubio, M. J.; Varas, J. (1997). El análisis de la realidad en la intervención social. Madrid: Ed. CCS.

Setién, M. L.(1993). Indicadores sociales de calidad de vida. Madrid: CIS.
Trilla, J. (coord.) (1997). Animación sociocultural. Teorías, programas y ámbitos. Barcelona, Ed. Ariel.

DireCCIÓN DE LA AUTORA: Belén Pascual Barrio. Universitat de les Illes Balears. Departamento de Pedagogía y Didácticas específicas. Ctra. Valldemossa, km. 7,5. 07122 Palma de Mallorca. Correo electrónicos: belen.pascual@uib.es

Fecha de recepción del artículo: 14.IX.2006

Fecha de aceptación definitiva: 19.X.2006 